\title{
Vitamin A rewrites the ABCs of oral tolerance
}

\author{
W Strober ${ }^{1}$
}

One of the chief requirements of an immune system, the mucosal immune system, that lies juxtaposed to a mass of potentially immunogenic commensal organisms is a well-developed mechanism to limit or negatively regulate nascent immune responses to those organisms. This mechanism, long subsumed under the name oral tolerance, is now understood to consist of a complex of factors, not the least of which is the ready ability to induce immunosuppressive regulatory T cells or Tregs. The emphasis here is on the "ability to induce" because the real individuality of the mucosal regulatory response lies not in the Tregs themselves, which after all can be induced anywhere and are mere tools of regulatory response. Now, as shown initially by the fact that oral tolerance is dependent on the size and mobility of its dendritic cell (DC) population, the individuality of the mucosal immune system is inherent in its inducing cells, i.e., the antigen-presenting DCs (or macrophages) of the mucosal immune system. ${ }^{1,2}$ Recently, new data have emerged that provide much more specific information on how mucosal DCs (or macrophages) are different in this respect and thus why they have a special tendency to facilitate the development of Tregs that then mediate oral tolerance. This is the subject of this brief review. The unresponsiveness of mucosal immune system to mucosal antigens is due to a process known as oral tolerance. Recent studies addressing the mechanism of such tolerance show that mucosal tissues are replete with a unique subset of dendritic cells that secrete factors such as, TGF- $\beta 1$ and retinoic acid, that induce foxp $3+$ regulatory $T$ cells. Thus, we arrive at the somewhat surprising realization that mucosal unresponsiveness is, appropriately enough, related to the availability of a factor in the food stream.

First, a bit of background: For some time now, information has been accumulating that supports the idea that certain mucosal dendritic cell (DC) subsets have a greater tendency than cells of the same subset in other tissues to produce cytokines that are known as regulatory cytokines rather than effector cytokines.
One of the earliest is a study by Iwasaki and Kelsall ${ }^{3}$ showing that CD11b + mucosal DCs produce far more interleukin (IL)-10 and far less IL-12p70 than spleen CD11b + DCs and probably more transforming growth factor (TGF)- $\beta 1$ as well. Similarly, in later studies it was shown that bronchial DCs had a special ability to produce IL-10, perhaps under the influence of ICOS costimulation, and recently it has been reported that immature mucosal DCs bear membrane TGF- $\beta 1{ }^{4,5}$ The reason why mucosal DCs are "different" from spleen DCs in this way is unclear, although there is some evidence that their physical association with epithelial cells may play some role. For instance, epithelial cells have been shown to produce cytokines that could affect DC development, such as thymic stromaderived lymphopoietin, a cytokine that directs DCs to induce Th 2 responses. ${ }^{6}$ In addition, epithelial cells produce IL-10, a cytokine that could act on DCs to inhibit IL-12p70 production and thus favor a shift from IL-12 to IL-10 production. ${ }^{7}$ Finally, and perhaps most importantly, epithelial cells produce TGF- $\beta 1$ and the large amount of this cytokine found in the intestine could be coming largely from this source. ${ }^{8,9}$ Whatever be the provenance of the special ability of mucosal DCs to produce regulatory cytokines, the latter have important downstream consequences: $T$ cells induced by $\mathrm{CD} 11 \mathrm{~b}+$ mucosal DCs tend to become Th2 2 cells ${ }^{3}$ or, if induced by DCs expressing cell-surface TGF- $\beta$ or producing TGF- $\beta$, they tend to become foxp $3+$ Tregs. ${ }^{5}$ Thus, in effect, the phenomenon of oral tolerance is to a large degree explained by the ability of specialized mucosal DCs to induce regulatory $\mathrm{T}$ cells.

Immediate precursor findings: The new evidence relating to interactions between DCs and oral tolerance provides further insight into the mechanisms of DC induction of Tregs. Two lines of research facilitated these studies. The first consisted of a large body of recent work showing that while foxp 3 + Tregs develop "centrally" in the thymus under the influence of IL-2,

${ }^{1}$ Mucosal Immunity Section, Laboratory of Host Defenses, National Institute of Allergy and Infectious Diseases, National Institutes of Health, Bethesda, Maryland, USA. Correspondence: W Strober (wstrober@niaid.nih.gov) 
such cells are also derived from nonregulatory (naive) $\mathrm{T}$ cells in the periphery by activation of the $T$ cells in the presence of TGF- $\beta 1$. In practice, this was assumed to occur during antigen-driven stimulation of the $\mathrm{T}$ cells by antigen-presenting cells (DCs) producing TGF- $\beta 1 .{ }^{5,10}$ The second comprised studies showing that mucosal DCs require the processing and secretion of retinoic acid (RA) to induce $\mathrm{T}$ cells (and B cells) to express surface markers (such as $\alpha_{\mathrm{E}} \beta_{7}$ and CCR9) that are essential for the mucosal cell trafficking that defines the common mucosal immune system. ${ }^{11-13}$ Thus, the following question arose: What is the combined role of TGF- $\beta 1$ and RA in the induction of Tregs in mucosal tissues?

The findings themselves: In the initial study addressing this issue, Mucida et al. ${ }^{14}$ laid out the main features of the new findings with data showing that mucosal DCs do, in fact, induce foxp $3+$ Tregs via the production of TGF- $\beta 1$ but, such induction requires the coproduction of RA. They thus established the key point that RA signaling is a necessary feature of regulatory cell induction in the mucosal immune system. Two studies following soon after corroborated these findings and added important details. In one such study by Coombes et al., ${ }^{15}$ it was shown that mucosal CD103+ DCs induced foxp $3+$ Tregs, but while induction was greatly augmented by the addition of exogenous TGF- $\beta 1$, it occurred in the absence of exogenous TGF- $\beta 1$ and was inhibited by anti-TGF- $\beta 1$. In further studies, it was shown that while mucosal DC induction of foxp3+ Tregs in the presence of TGF- $\beta 1$ was not enhanced by the addition of RA, the addition of RA receptor inhibitors downregulated the TGF- $\beta 1$ effect. These studies are thus compatible with the view that mucosal CD103 + DCs are fully competent to induce foxp $3+$ Tregs when provided with exogenous TGF- $\beta 1$ because they already produce adequate amounts of RA. A somewhat different picture was obtained with CD103 - mucosal DCs. Here it was found that no induction of foxp $3+$ Tregs occurred unless exogenous TGF- $\beta 1$ was present and then the induction was relatively weak. On the other hand, the addition of both TGF- $\beta 1$ and
RA to this DC population was associated with induction of foxp $3+$ Tregs at a level equal to that of the CD103+ DCs. Finally, while CD103 - DCs were poor inducers of foxp $3+$ Tregs (in the absence of exogenous TGF- $\beta 1$ and RA), they were excellent producers of effector cytokines. The authors speculated that the difference between CD103 + and CD103 - mucosal DCs is that the presence of CD103 on DCs facilitates DC interactions with epithelial cells, possibly through E-cadherin, a known ligand of CD103. This then leads to acquisition of the ability to upregulate the enzymes necessary for the production of RA as well as the ability to produce and/or activate TGF- $\beta 1$. CD103- DCs do not participate in such epithelial cell interactions and thus do not acquire these characteristics.

A second study conducted by Sun et al. reached much the same conclusions. ${ }^{16}$ In these studies, it was shown with cells from mice with a GFP cassette inserted into the foxp $3+$ site that thus express easily identifiable GFP whenever they express foxp 3 + that the mesenteric lymph node and lamina propria (especially the Peyer's patches) are a "hot bed" of conversion of naive cells to foxp 3 + cells in the context of antigen stimulation. Then, in studies of induction of foxp 3 + Tregs by lamina propria DCs, it was again shown that while CD103 + but not CD103 - DCs induce Tregs in the absence of TGF- $\beta 1$, both populations have this property in the presence of TGF- $\beta 1$ (even in the absence of exogenous RA). On the other hand, spleen DCs were poor inducers of such Tregs. Finally, the addition of RA also enhanced CD103 + DC and CD103 - DC induction of Tregs above that seen with TGF- $\beta 1$ alone, and an inhibitor or RA signaling abolishes the inductive effect of TGF- $\beta 1$. In these studies as in the studies by Coombes et al., ${ }^{15} \mathrm{CD} 103-$ DCs are good inducers of foxp $3+$ Tregs, provided exogenous TGF- $\beta 1$ and RA are present. The authors of this paper emphasized that vitamin $A$ is a component of food, and its conversion to RA at this site is facilitated by the presence of appropriate enzymes in the epithelia and perhaps other mucosal cells. In this view, the availability of an RA precursor (vitamin A) is a unique aspect of the mucosal milieu and one that ultimately lies behind the abundance of Tregs produced at this site.

Yet another study by Denning et al. ${ }^{17}$ relates lamina propria antigen-presenting cells to suppressor cell induction. However, in this case, the inducing cells are macrophages rather than DCs and IL-10 is shown to be an important inductive factor in addition to TGF- $\beta 1$ and RA. Thus, while these findings again posit that mucosal antigen-presenting cells producing TGF- $\beta 1$ and RA are inducers of Tregs, the inducing cells in this study are very different and IL-10 is added to the mix of inducing cytokines. Further work will be necessary to resolve these differences.

New questions: The above body of work provides impressive evidence supporting the importance of RA in the induction of mucosal immune regulatory function. Yet, as in the case of every advance, they bring into view a set of new questions. First, of course, is the question of the molecular basis of RA function in regulatory T-cell development. One key fact is already evident: RA acts in concert with TGF- $\beta 1$ to induce regulatory cells and has little or no effect on its own. This means that it somehow enhances whatever signaling pathway set in motion by TGF- $\beta 1$ that is responsible for the induction of foxp3 expression and whatever other cellular changes that occur to obtain a functioning regulatory cell. Previous studies have shown that RA actually impedes Smad function, the main TGF- $\beta 1$ signaling pathway in some hematopoietic cells; ${ }^{18}$ thus, its effects on TGF- $\beta 1$ signaling in this case is somewhat paradoxical. Given the fact that several cytokines have been shown to negatively affect TGF- $\beta 1$ induction of Tregs, such as IL-6 and IL-27, it is also possible that RA works to inhibit certain natural inhibitory pathways. Finally, it is also possible that RA affects negative or reinforces positive effects of $\mathrm{T}$ cell costimulation on Treg induction.

Another but related question concerns the source of RA and the factors leading to the acquisition of the enzyme systems necessary for the conversion of RA precursors to RA. There is some evidence that DCs are one such source, but other sources may also be present. Certainly, the mucosal epithelial cell must be considered in this light. 


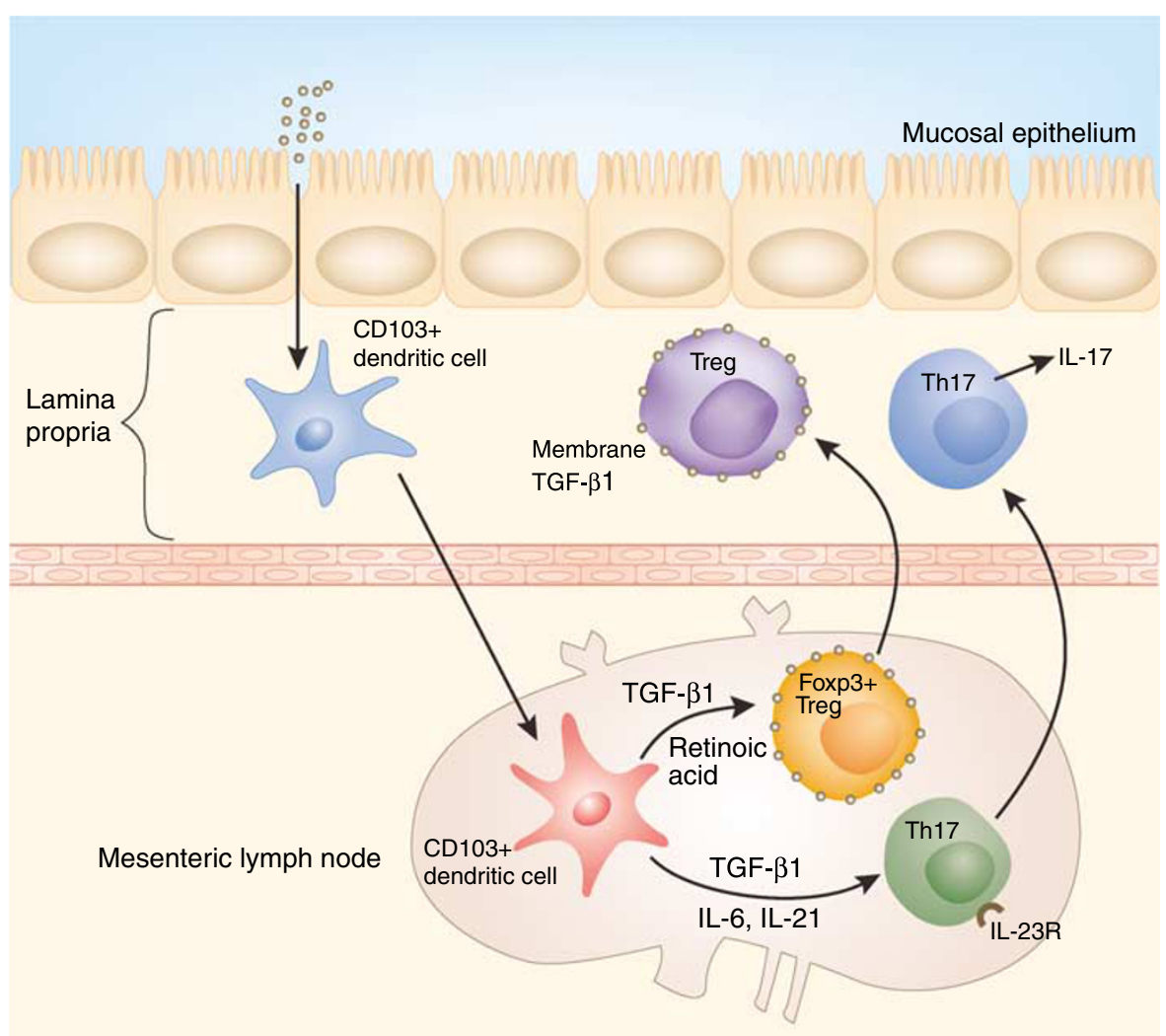

Figure 1 CD103 + DCs in the lamina propria of the gut can reciprocally induce either Th17 or Tregs. Upon secretion of TGF- $\beta 1$ and retinoic acid, DCs induce Tregs; alternatively, upon secretion of TGF- $\beta 1$ and IL-6/IL-21, DCs induce Th17 cells. TGF- $\beta 1$ appears on the surface of Tregs and IL-23R appears on the surface of Th17 cells. Both Tregs and Th17 cells traffic back to the lamina propria. DC, dendritic cell; TGF- $\beta 1$, transforming growth factor- $\beta 1$.

A third question relates to an important finding by the first study addressing the role of RA, mentioned above, that of Mucida et al. ${ }^{14}$ These investigators showed that RA mediates an important reciprocality between the Treg induction and Th17 induction: DCs stimulated in the presence of TGF- $\beta 1$ induce Tregs in the presence of RA and induce Th17 cells in the absence of RA (and the presence of IL-6). Thus, RA governs whether the mucosal system will generate an effector cell or regulatory cell response. Recent studies of Th17 differentiation have shown that it is a two-stage process involving, first, the induction of a lineage-specific factor, retinoic acid orphan receptor $\gamma \mathrm{T}(\mathrm{ROR} \gamma \mathrm{t})$ and then the induction of IL- 17 by ROR $\gamma$ t. ${ }^{19}$ TGF- $\beta 1$ has been shown to act at the level of ROR $\gamma \mathrm{t}$ induction, as does IL-6 through Stat3. In murine systems, IL-23 appears to act as a secondary player that comes into play as an expander of nascent Th17 cells after IL- 6 induces IL- 21 and the latter induces
IL-23R expression. ${ }^{20}$ The reciprocality between Th17 induction and Treg induction mentioned above strongly implies that foxp3, induced as a result of RA secretion, acts as a molecular switch that on the one hand retards ROR $\gamma t$ transcriptional activity and on the other induces Treg transcriptional activity (Figure 1). Studies currently being conducted by a group headed by Littman and his colleagues provide data supporting this view (personal communication). Nevertheless, the question of just how RA functions at a molecular level to decide the outcome remains unanswered. One possibility that needs to be investigated is whether Th17 or alternative Treg differentiation is determined by the quality or quantity of the TGF- $\beta 1$ signal and this, in turn, is decided by the RA cosignal.

Other questions that remain include those raised by Denning et al., mentioned above, addressing the role of macrophages and IL-10 in regulatory T-cell development in the mucosa. ${ }^{15}$ This is important, if only because IL-10-dependent (Tr-1) Tregs play a significant role in oral tolerance as well as foxp $3+$ Tregs. ${ }^{21}$ Thus, while knowledge of mucosal tolerance and the generation of regulatory cells in the mucosa has reached a new plateau, new research territory is now in sight; the mucosal beat goes on.

\section{DISCLOSURE}

The author declared no conflict of interest.

(C) 2008 Society for Mucosal Immunology

\section{REFERENCES}

1. Viney, J.L. et al. Expanding dendritic cells in vivo enhances the induction of oral tolerance. J. Immunol. 160, 5815-5825 (1998).

2. Worbs, T. et al. Oral tolerance originates in the intestinal immune system and relies on antigen carriage by dendritic cells. J. Exp. Med. 203, 519-527 (2006).

3. Iwasaki, I. \& Kelsall, B.L. Freshly isolated Peyer's patch, but not spleen, dendritic cells produce interleukin 10 and induce the differentiation of $\mathrm{T}$ helper type 2 cells. J. Exp. Med. 190, 229-239 (1999).

4. Akbari, O. et al. Antigen-specific regulatory T cells develop via the ICOS-ICOS-ligand pathway and inhibit allergen-induced airway hyperreactivity. Nat. Med. 8, 1024-1032 (2002).

5. Gandhi, R. et al. Cutting Edge: Immature human dendritic cells express latency-associated peptide and inhibit T cell activation in a TGF- $\beta$ dependent manner. J. Immunol. 178, 4016-4021 (2007).

6. Rimoldi, M. et al. Intestinal immune homeostasis is regulated by the crosstalk between epithelial cells and dendritic cells. Nat. Immunol. $\mathbf{6}$, 507-514 (2005).

7. Autschbach, F. et al. In situ expression of interleukin-10 in noninflamed human gut and in inflammatory bowel diseases. Am. J. Pathol. 153, 121-130 (1998)

8. Boirivant, M. et al. Inhibition of Smad7 with a specific antisense oligonucleotide facilitates TGF- $\beta 1$-mediated suppression of colitis. Gastroenterology 131, 1786-1798 (2006).

9. Becker, C. et al. TGF- $\beta$ as a T cell regulator in colitis and colon cancer. Cytokine Growth Factor Rev. 17, 97-106 (2006).

10. Marie, J.C. et al. TGF- $\beta$ maintains suppressor function and Foxp3 expression in CD4+ CD25+ regulatory T cells. J. Exp. Med. 201, 1061-1067 (2005).

11. Mora, J.R. et al. Generation of gut-homing lgAsecreting $B$ cells by intestinal dendritic cells. Science 314, 1157-1160 (2006).

12. Iwata, M. et al. Retinoic acid imprints gut-homing specificity on T cells. Immunity 21, 527-538 (2004).

13. Svensson, M. et al. Retinoic acid receptor signaling levels and antigen dose regulate gut homing receptor expression on $\mathrm{CD} 8^{+} \mathrm{T}$ cells. Mucosal Immunol. 1, 38-48 (2008).

14. Mucida, D. et al. Reciprocal $T_{H} 17$ and regulatory $T$ cell differentiation mediated by retinoic acid. Science 317, 256-260 (2007)

15. Coombes, J. et al. A functionally specialized population of mucosal CD103+ DCs induces Foxp3+ regulatory T cells via a TGF- $\beta$ - and 
retinoic acid-dependent mechanism. J. Exp. Med. 204, 1757-1764 (2007).

16. Cheng-Ming, S. et al. Small intestine lamina propria dendritic cells promote de novo generation of Foxp3 T reg cells via retinoic acid. J. Exp. Med. 204, 1775-1785 (2007).

17. Denning, T. et al. Lamina propria macrophages and dendritic cells differentially induce regulatory and interleukin 17-producing T cell responses. Nat. Immunol. 8, 1086-1094 (2007).

18. Cao, Z. et al. Levels of phosphor-Smad2/3 are sensors of the interplay between effects of TGF- $\beta$ and retinoic acid on monocytic and granulocytic differentiation of HL-60 cells. Blood 10, 498-507 (2003).

19. Ivanov, I.I. et al. The orphan nuclear receptor ROR $\gamma$ t directs the differential program of proinflammatory IL-17+ Thelper cells. Cell 126, 1121-1133 (2006).

20. Zhou, L. et al. IL-6 programs $\mathrm{TH}-17$ cell differentiation by promoting sequential engagement of the IL-21 and IL-23 pathways. Nat. Immunol. 8, 967-974 (2007).

21. Battaglia, M. et al. IL-10 producing T regulatory type 1 cells and oral tolerance. Ann. N.Y. Acad. Sci. 1029, 142-153 (2004). 